\title{
EXTINCTION RADIAL PROFILES OF M83 FROM GALEX ULTRAVIOLET IMAGING
}

\author{
Samuel Boissier, ${ }^{1}$ Armando Gil de Paz, ${ }^{1}$ Barry F. Madore, ${ }^{1}$ Alessandro Boselli, ${ }^{2}$ Véronique Buat, ${ }^{2}$ \\ Denis Burgarella, ${ }^{2}$ Peter G. Friedman, ${ }^{3}$ Tom A. Barlow, ${ }^{3}$ Luciana Bianchi, ${ }^{4}$ Yong-Ik Byun, ${ }^{5}$ José Donas, ${ }^{2}$ \\ Karl Forster, ${ }^{3}$ Timothy M. Heckman, ${ }^{6}$ Patrick N. Jelinsky, ${ }^{7}$ Young-Wook Lee, ${ }^{5}$ Roger F. Malina, ${ }^{2}$ \\ D. Christopher Martin, ${ }^{3}$ Bruno Milliard, ${ }^{2}$ Patrick Morrissey, ${ }^{3}$ Susan G. Neff, ${ }^{8}$ R. Michael Rich, ${ }^{9}$ \\ David Schiminovich, ${ }^{3}$ Oswarld H. W. Siegmund, ${ }^{7}$ Todd Small, ${ }^{3}$ Alex S. Szalay, ${ }^{6}$ \\ BARRY Y. WELSH, ${ }^{7}$ AND TED K. WYDER ${ }^{3}$ \\ Received 2004 April 20; accepted 2004 June 24; published 2005 January 17
}

\begin{abstract}
We use the far-UV (FUV) and near-UV images of M83 obtained by the Galaxy Evolution Explorer (GALEX) to compute the radial profile of the UV spectral slope in the star-forming disk. We briefly present a model of its chemical evolution, which allows us to obtain realistic intrinsic properties of the stellar populations. Using corollary data, we also compute the profiles of $\mathrm{H} \alpha / \mathrm{H} \beta$ and total-IR/FUV ratios. Both the data and model are used to estimate and compare the extinction gradients at the FUV wavelength obtained from these various indicators. We discuss the implications for the determination of the star formation rate.
\end{abstract}

Subject headings: dust, extinction — galaxies: individual (M83) — ultraviolet: galaxies

Online material: color figures

\section{INTRODUCTION}

UV and optical observations of galaxies are, in general, affected by dust extinction. Empirical determinations of the star formation rate in individual galaxies, modeling of the radial properties and color gradients of galaxies (e.g., MacArthur et al. 2004), or estimations of the "cosmic star formation rate history" (e.g., Madau et al. 1998) all need to take into account its effects. While detailed radiative transfer calculations selfconsistently accounting for the whole spectral energy distribution (SED) can be used to estimate this effect (e.g., Popescu et al. 2000, 2004), it is usually done by applying much simpler standard recipes.

These recipes include the following:

1. Balmer decrement method.-This involves a comparison of the observed $\mathrm{H} \alpha / \mathrm{H} \beta$ ratio with its predicted value (2.86 for case B recombination; e.g., Osterbrock 1989). Although usually

\footnotetext{
${ }^{1}$ Observatories of the Carnegie Institution of Washington, 813 Santa Barbara Street, Pasadena, CA 91101; boissier@ociw.edu, agpaz@ociw.edu, madore@ ociw.edu.

${ }^{2}$ Laboratoire d'Astrophysique de Marseille, BP 8, Traverse du Siphon, 13376 Marseille Cedex 12,France; alessandro.boselli@oamp.fr, veronique.buat@oamp.fr, denis.burgarella@oamp.fr, jose.donas@oamp.fr, roger.malina@oamp.fr, bruno .milliard@oamp.fr.

${ }^{3}$ California Institute of Technology, MC 405-47, 1200 East California Boulevard, Pasadena, CA 91125; friedman@srl.caltech.edu, tab@srl.caltech.edu, krl@srl.caltech.edu,cmartin@srl.caltech.edu,patrick@srl.caltech.edu,ds@srl .caltech.edu, tas@srl.caltech.edu,wyder@srl.caltech.edu.

${ }^{4}$ Center for Astrophysical Sciences, Johns Hopkins University, 3400 North Charles Street, Baltimore, MD 21218; bianchi@skysrv.pha.jhu.edu.

${ }^{5}$ Center for Space Astrophysics, Yonsei University, Seoul 120-749, Korea; byun@csa.yonsei.ac.kr, ywlee@csa.yonsei.ac.kr.

${ }^{6}$ Department of Physics and Astronomy, Johns Hopkins University, Homewood Campus, Baltimore, MD 21218; heckman@pha.jhu.edu, szalay@pha .jhu.edu.

${ }^{7}$ Space Sciences Laboratory, University of California at Berkeley, 601 Campbell Hall, Berkeley, CA 94720; patj@ssl.berkeley.edu, ossy@ssl.berkeley.edu, pwelsh@ssl.berkeley.edu.

${ }^{8}$ Laboratory for Astronomy and Solar Physics, NASA Goddard Space Flight Center, Greenbelt, MD 20771; neff@stars.gsfc.nasa.gov.

${ }^{9}$ Department of Physics and Astronomy, University of California, Los Angeles, CA 90095; rmr@astro.ucla.edu.
}

used for $\mathrm{H} \alpha$ attenuation, its results can be extrapolated to other wavelengths (e.g., Buat et al. 2002).

2. UV spectral slope method.-Meurer et al. (1999, hereafter M99) proposed a relation between the UV extinction and the UV spectral slope $\beta$ (the continuum spectrum $f_{\lambda}$ between 1300 and $2600 \AA$, having a shape of $\lambda^{\beta}$ ) in starbursts. Recently, Kong et al. (2004, hereafter K04) computed the equivalent relationship in the Galaxy Evolution Explorer (GALEX) bands.

3. The total-IR (TIR)/UV luminosity ratio method (see M99).Radiative transfer models have shown that this ratio is a robust indicator of the UV extinction, since it does not depend much on either the geometry or the star formation history (Gordon et al. 2000)

Each of the above methods has its own limitations. The Balmer decrement method is affected by systematics caused by uncertainties in the underlying absorption of the stellar populations, and by the fact that it concerns only very massive stars and may not represent the extinction affecting older stars. The UV spectral slope method was calibrated for starburst galaxies and may not apply more generally. Moreover, both methods rely on colors determined at similar wavelengths that are quite sensitive to dust radiative transfer effects (Witt et al. 1992; Witt \& Gordon 2000, hereafter WG00). Finally, the TIR/UV method is the most reliable, but until the advent of the Infrared Space Observatory (ISO) and now the Spitzer Space Telescope, this method was limited in its application by the low spatial resolution of the IRAS data.

M83 is an interesting laboratory to test the recipes for extinction, given the profusion of data available in addition to the GALEX images, and for being almost face-on. Models of its stellar content and chemical evolution can be constrained by these data and used to predict the intrinsic properties of the stellar population. An alternative study combining observations of M101 from GALEX and ISO is presented in Popescu et al. (2005).

\section{OBSERVATIONS}

GALEX observed M83 for a total of 1352 s on 2003 June 7 simultaneously through its far-UV (FUV) and near-UV (NUV) 

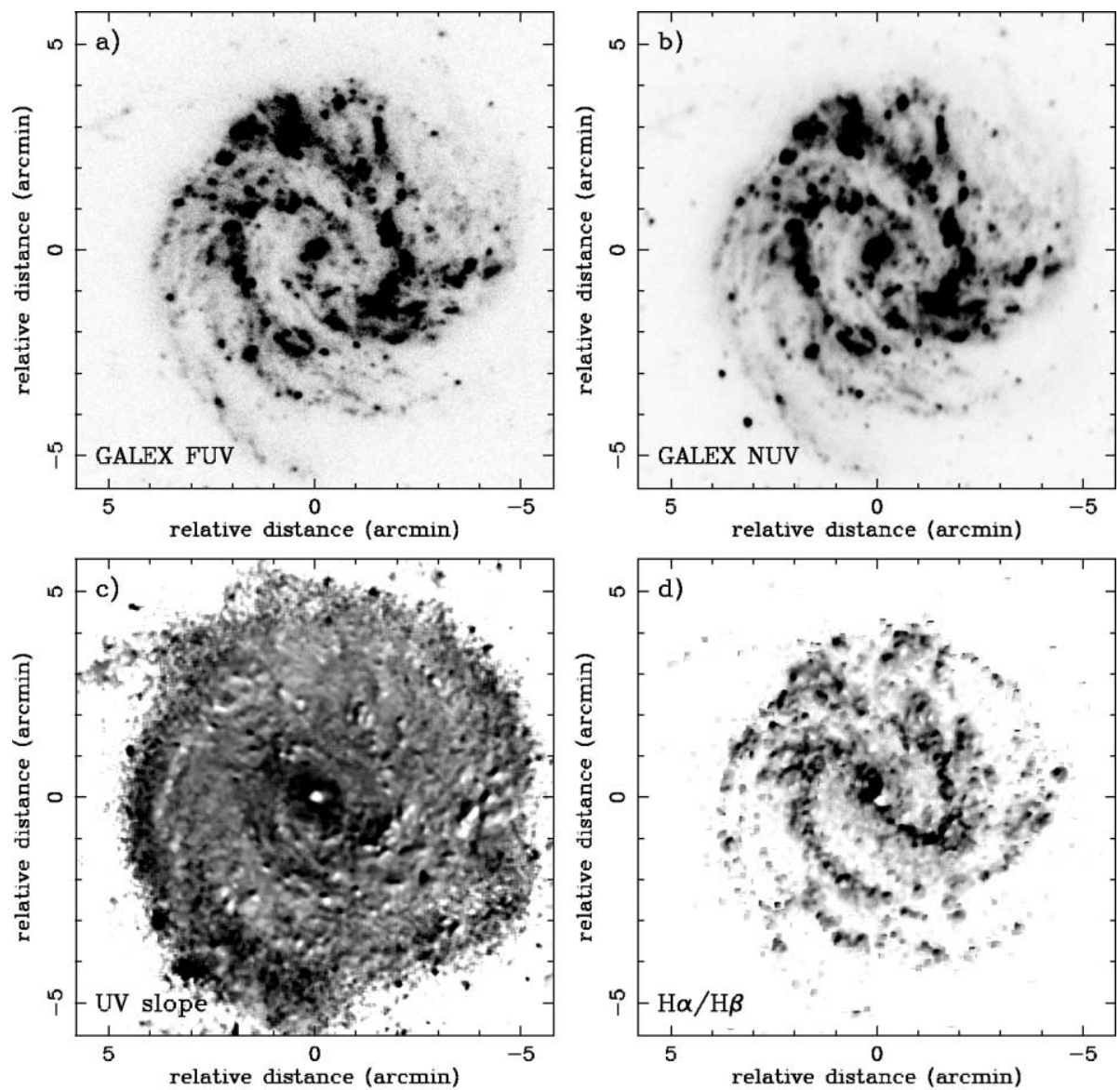

FIG. 1.-(a) FUV image (from the sky background to $22.3 \mathrm{AB}$ mag $\operatorname{arcsec}^{-2}$ ). (b) NUV image (from the sky background to $22.7 \mathrm{AB}$ mag arcsec ${ }^{-2}$ ). (c) UV spectral slope $\beta$, from -2.5 (white) to 0.3 (black). (d) $\mathrm{H} \alpha / \mathrm{H} \beta$ ratio, from $\mathrm{H} \alpha / \mathrm{H} \beta=2$ to 6 . White areas in this plot correspond to regions where either $\mathrm{H} \alpha$ or $\mathrm{H} \beta$ was below $3 \sigma_{\text {sky. }}$. A $8^{\prime \prime} \times 8^{\prime \prime}$ median filter was applied in the cases of $(c)$ and $(d)$.

bands (see Fig. 1). From these images, we derive the FUV and NUV profiles by azimuthally averaging along ellipses after masking the stars and subtracting the sky. The ellipticity and position angle $\left(0.1\right.$ and $\left.80^{\circ}\right)$ were taken from Kuchinski et al. (2000). In this paper, the authors presented Ultraviolet Imaging Telescope (UIT) observations of M83. The galaxy was observed by UIT only in the FUV domain, and with a slightly better resolution $\left(\sim 3^{\prime \prime}\right)$ than the GALEX one $\left(\sim 5^{\prime \prime}\right)$. The surface brightness sensitivity limit of the GALEX image, however, is $\sim 2$ mag deeper. Our FUV profile is in good agreement with the one published in Kuchinski et al. (2000), but extends to larger radii.

The map and radial profile of the UV spectral slope $\beta_{G L X}$ (computed in the GALEX bands as in K04) are shown in Figures $1 c$ and $2 a$, respectively. We estimate $0.15 \mathrm{mag}$ to be the uncertainty in the calibration of the GALEX $\mathrm{AB}$ surface brightnesses.

We derive the $\mathrm{H} \alpha$ and $\mathrm{H} \beta$ profiles from narrowband imaging $(\mathrm{FWHM} \simeq 60 \AA$ ) in the lines and adjacent continuum images, all obtained at the Las Campanas Observatory (LCO; Chile) 40 inch $(1.0 \mathrm{~m})$ telescope using the $2048 \times 3150$ pixel CCD camera.

To estimate the $[\mathrm{N}$ II] $\lambda \lambda 6548,6584$ contamination of the $\mathrm{H} \alpha$ image and the underlying Balmer absorption, we used a drift-scanning long-slit spectrum of M83 obtained with the WFCCD spectrograph at the LCO 100 inch $(2.5 \mathrm{~m})$ telescope. We measured $[\mathrm{N}$ II $] / \mathrm{H} \alpha=0.45$. Both $\mathrm{H} \beta$ absorption and emission were detected along the central $\pm 4^{\prime}$ of M83. The average underlying absorption at $\mathrm{H} \beta$ is $4.2 \AA$ ( $\mathrm{rms}=1 \AA$ ). Since the absorption in $\mathrm{H} \alpha$ could not be measured, we assumed it to be the same as for $\mathrm{H} \beta$ (e.g., McCall et al. 1985).

Profiles were computed from the 60 and $100 \mu \mathrm{m}$ images obtained by a IRAS-HIRES request (Rice 1993). The $60 \mu \mathrm{m}$ image was convolved with a Gaussian to obtain a resolution similar to the $100 \mu \mathrm{m}$ image $\left(\sim 90^{\prime \prime}\right)$. The two profiles were then combined to compute the TIR surface brightness profile, following Dale et al. (2001). The resolution of the FUV images was degraded in a similar way, before computing the TIR/FUV ratios profiles.

\section{MODELS OF M83}

We use models for the chemical and spectrophotometric evolution of M83 based on Boissier \& Prantzos (2000, hereafter BP00), with an updated star formation law (depending on the angular velocity) determined in Boissier et al. (2003). To compute the star formation rate (SFR), we adopt the rotation curve of Crosthwaite et al. (2002). These models, however, cannot reproduce the observed gaseous profile (taken from Crosthwaite et al. 2002; Lundgren et al. 2004). This is most likely because the models do not include radial inflows. There are reasons why such a flow might be present in M83: the strong bar could induce radial motion, and the central starburst needs to be supplied with fresh gas. Interaction with NGC 5253 may be responsible for these peculiarities (van den Bergh 1980). We introduce in the models of BP00 radial inflows of various 


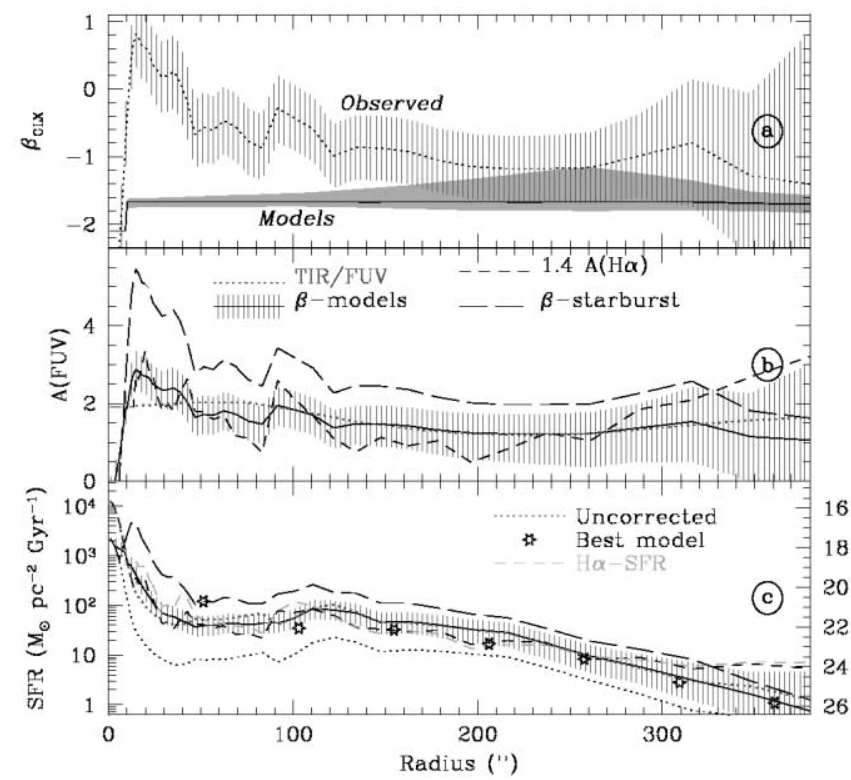

FIG. 2.-(a) Radial profile of the observed UV spectral slope (dotted line, derived from the GALEX bands). The solid curve (shaded area) indicates at each radius the average $\beta_{G L X}$ (the range of values) obtained with models providing satisfactory profiles. (b) FUV extinction profiles derived from various indicators (see legend in the figure and details in text). (c) FUV-deduced SFR profiles (surface brightness scale on the right in mag $\operatorname{arcsec}^{-2}$ ), uncorrected for extinction (dotted line) and with the extinction corrections given in $(b)$. The stars show the current SFR obtained with the model. We also show the SFR deduced from $\mathrm{H} \alpha$, corrected for $A(\mathrm{H} \alpha)$. [See the electronic edition of the Journal for a color version of this figure.]

efficiencies and various starting times. The best models chosen are the ones reproducing the observed dust-free profiles: total gas and near-infrared surface brightness of the Two Micron All Sky Survey. Several models that include a flow beginning a few gigayears ago $(1-5 \mathrm{Gyr})$ produce satisfactory results. In Figure $2 a$, we show the profile of the average intrinsic spectral UV slope ( $\beta_{0}$, short-dashed curve), and the range of values (shaded area) obtained with these best models. ${ }^{10}$

\section{EXTINCTION MAPS AND PROFILES}

We now consider the "classical" extinction estimators used in extragalactic studies:

1. The $A(\mathrm{H} \alpha)$ extinction obtained from the $\mathrm{H} \alpha / \mathrm{H} \beta$ ratio (after correction for the Balmer underlying absorption) can be extrapolated to the FUV wavelength. We obtain $A($ FUV $)=$ $1.4 A(\mathrm{H} \alpha)$ by assuming that the color excess of the stellar continuum is 0.44 times that of the gaseous emission (see Buat et al. 2002 and references within), and using the Galactic extinction curve of Pei (1992), for which $A(\mathrm{H} \alpha) / E(\mathrm{H} \beta-$ $\mathrm{H} \alpha)=2.26$.

2. It is common to apply the method of M99 in galaxies with nuclear starbursts like M83. We compute a profile following this assumption ( $\beta$-starburst, Fig. $2 b$ ), adopting the TIR/ FUV- $\beta_{G L X}$ relation computed by K04.

3. Finally, we compute the extinction $A(\mathrm{FUV})$ from the TIR/ FUV ratio, following Buat et al. (2005). The result is presented

\footnotetext{
${ }^{10}$ In the central $50^{\prime \prime}$ of the galaxy, the presence of the starburst and a bulge are not taken into account in the disk model. In the central starburst itself $\left(10^{\prime \prime}\right)$, the UV spectral slope of the model is artificially changed to the canonical value -2.1 used for the intrinsic slope of a starburst (Calzetti et al. 2000). More details on the models will be given in a forthcoming paper.
}
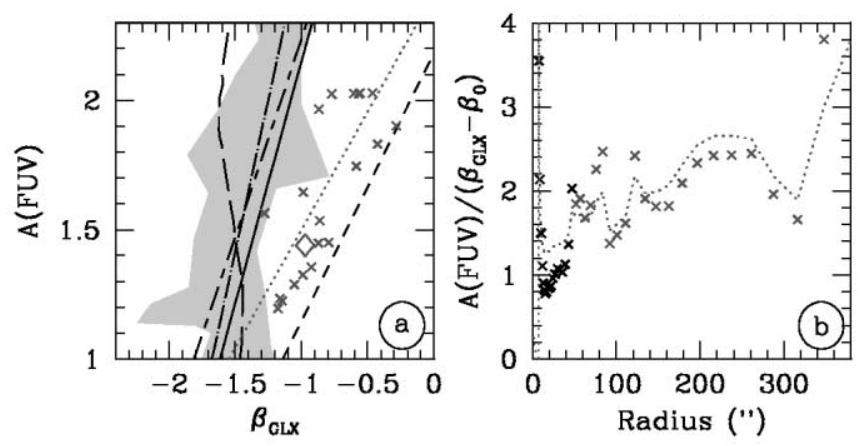

FIG. 3.-Relation between FUV extinction and UV spectral slope. (a) Crosses show the FUV extinction ( $A[F U V]$ from the TIR/FUV ratio) as a function of $\beta_{G L X}$ for our data out of the bulge (i.e., the central $50^{\prime \prime}$ ). The dotted line is the least-squares fit. The shaded area includes the integrated values found by Buat et al. (2005). The short-long-dashed line is the relation found for starbursts in K04. The solid curve is obtained with the model of K04, for a present-to-past averaged star formation $(b)$ such that $\log b=0.2$, a typical value obtained with our model of M83. The short- and long-dashed curves show the relation expected for different geometrical models of WG00 (respectively shell-homogeneous and dusty-clumpy). (b) FUV extinction to $\left(\beta-\beta_{0}\right)$ ratio as a function of radius (crosses). The dotted curve is obtained using the previous fit instead of $A(\mathrm{FUV})$. [See the electronic edition of the Journal for a color version of this figure.]

in Figure $2 b$. The absence of features in this gradient is due to the low-resolution infrared IRAS observations. To achieve a better spatial resolution that takes advantage of the TIR/FUV calibration for $A(\mathrm{FUV})$, we perform a fit (outside the 50 central arcseconds of the bulge) providing $A(\mathrm{FUV})=0.86+0.91$ $\left(\beta_{G L X}-\beta_{0}\right)$ (Fig. 3, dotted lines, shifted to account for the average value of $\beta_{0}$ ). We use this result to compute a new extinction profile ( $\beta$-models in Fig. 2 , middle) from the $\beta_{G L X}$ profile.

The extinction maps (Fig. 1) and the profiles (Fig. 2) show that the extinction is relatively low in the very center (see the hole at the center of the $\beta_{G L X}$ map, Fig. 1c), assuming that the low values of $\beta_{G L X}$ in this region indicate low extinction. The extinction (indicated by the various tracers) becomes rapidly high in a circumnuclear region and progressively decreases as we move into the disk of the galaxy. The various indicators show some common structure: the spectral slope $\beta_{G L X}$ (and the extinction profiles derived from it), in addition to the Balmer decrement, show several correlated peaks. One of the most remarkable peaks is located $100^{\prime \prime}$ from the center. Inspection of the images (Fig. 1) shows that this radius corresponds to the position of spiral arms where both the star formation rate and the extinction are enhanced with respect to average regions of the galaxy. The $\beta$-starburst extinction gives larger values than other indicators; i.e., M83 shows lower extinctions (computed from the TIR/FUV ratio) than starburst galaxies for the same value of $\beta$. This is in agreement with the deviations with respect to the starburst case already obtained in the LMC (Bell 2002) or in less active galaxies (K04).

We used a purely empirical relation above for $A(\mathrm{FUV})$ and $\left(\beta_{G L X}-\beta_{0}\right)$ in M83. Because of the many uncertainties (geometry, $\beta_{0}$, etc.), we prefer this to theoretical prescription. In Figure $3 a$, we compare our data outside the bulge with two models of WG00 (adopting for $\beta_{0}$ the value of our best model) to illustrate the dependence on the geometry. We also compare our results with the model of $\mathrm{K} 04$ for the birthrate parameter $b$ obtained in our model of M83 $(\log b \simeq 0.2)$. We find that it overpredicts $A(\mathrm{FUV})$ by a large amount for any given value of $\beta_{G L X}$. Finally, our data are compared to other samples: the 
fit of K04 for the starbursts of M99 (solid line) and the integrated NUV- and TIR-selected galaxies of Buat et al. (2005). The profile of M83 is consistent with the latter, although with slightly larger $\beta_{G L X}$. Figure $3 b$ shows the $A(\mathrm{FUV}) /\left(\beta-\beta_{0}\right)$ ratio (in which $A[\mathrm{FUV}]$ is still determined from the TIR/FUV ratio) as a function of radius [the dotted curve indicates the same profile if the extinction derived from $\left(\beta-\beta_{0}\right)$ as discussed previously is used instead of the TIR/FUV ratio]. Outside the bulge, $A(\mathrm{FUV}) /\left(\beta-\beta_{0}\right)$ has an average value of 1.9 (this value would become 1.2 if the usual $\beta_{0}=-2.1$ were adopted).

\section{IMPLICATIONS FOR THE STAR FORMATION RATE}

The FUV surface brightness profile can be transformed into a star formation rate profile using the standard conversion factor of Kennicutt (1998): SFR $\left(M_{\odot} \mathrm{yr}^{-1}\right)=1.4 \times 10^{-28} L_{\mathrm{UV}}(\mathrm{ergs}$ $\left.\mathrm{s}^{-1} \mathrm{~Hz}^{-1}\right)$. Regardless of the uncertainties affecting this number (see Kennicutt 1998), the FUV profile must be corrected for extinction to obtain a reliable SFR. Figure $2 c$ shows the SFR profile obtained without extinction correction (lower dotted line) and with various corrections proposed in the previous section. ${ }^{11}$ We find that the SFR profile of our best model (stars, Fig. $2 c$ ) is in good agreement with the one deduced from the observations using the TIR/FUV correction. This suggests that the star formation law used in our evolutionary models is quite realistic.

\footnotetext{
${ }^{11}$ We also show the SFR profile deduced from the $\mathrm{H} \alpha$ surface brightness profile, corrected from $A(\mathrm{H} \alpha)$, and it is very similar to the SFR deduced from the FUV. This fact indicates that the current azimuthally averaged SFR is not
} very different from the SFR averaged over the last $10^{8} \mathrm{yr}$.
We can compute the SFR of M83 inside 300", according to various assumptions (adopting a distance of $4 \mathrm{Mpc}$; de Vaucouleurs 1979). With no extinction correction, the derived star formation rate is $1 M_{\odot} \mathrm{yr}^{-1}$. Assuming the whole galaxy behaves like a starburst $\left(\beta\right.$-starburst), we obtain $10.1 M_{\odot} \mathrm{yr}^{-1}$. This assumption probably overestimates the star formation rate, since the starburst is located only in the center. The extinction based on the Balmer decrement gives a SFR of $3.3 M_{\odot} \mathrm{yr}^{-1}$, and the one based on a combination of the TIR/FUV ratio and $\beta_{G L X}$ profiles provides $3.6 M_{\odot} \mathrm{yr}^{-1}$ (a similar value of $4 M_{\odot} \mathrm{yr}^{-1}$ is obtained from the low-resolution TIR/FUV extinction profile).

To summarize, we used GALEX observations of M83 in combination with other data to compare several extinction indicators and to estimate the effect of extinction on the determination of the SFR. Differences of a factor up to 2.5 are obtained with the various methods. Moreover, we determined an extinction-free SFR profile that allows to check the validity of the SFR law used in evolutionary models. A similar analysis will be applied in a later paper to a larger number of galaxies observed by GALEX.

GALEX (Galaxy Evolution Explorer) is a NASA small explorer launched in 2003 April. We gratefully acknowledge NASA's support for construction, operation, and science analysis for the GALEX mission, developed in cooperation with the Centre National d'Etudes Spatiales of France and the Korean Ministry of Science and Technology. We thank C. Popescu and R. Tuffs for their comments. S. B. thanks the CNES for its financial support.

\section{REFERENCES}

Bell, E. F. 2002, ApJ, 577, 150

Boissier, S., \& Prantzos, N. 2000, MNRAS, 312, 398 (BP00)

Boissier, S., Prantzos, N., Boselli, A., \& Gavazzi, G. 2003, MNRAS, 346, 1215

Buat, V., Boselli, A., Gavazzi, G., \& Bonfanti, C. 2002, A\&A, 383, 801

Buat, V., et al. 2005, ApJ, 619, L51

Calzetti, D., Armus, L., Bohlin, R. C., Kinney, A. L., Koornneef, J., \& StorchiBergmann, T. 2000, ApJ, 533, 682

Crosthwaite, L. P., Turner, J. L., Buchholz, L., Ho, P. T. P., \& Martin, R. N. 2002, AJ, 123, 1892

Dale, D. A., Helou, G., Contursi, A., Silbermann, N. A., \& Kolhatkar, S. 2001, ApJ, 549, 215

de Vaucouleurs, G. 1979, AJ, 84, 1270

Gordon, K. D., Clayton, G. C., Witt, A. N., \& Misselt, K. A. 2000, ApJ, 533, 236

Kennicutt, R. C., Jr. 1998, ARA\&A, 36, 189

Kong, X., Charlot, S., Brinchmann, J., \& Fall, S. M. 2004, MNRAS, 349, 769 (K04)

Kuchinski, L. E., et al. 2000, ApJS, 131, 441
Lundgren, A. A., Wiklind, T., Olofsson, H., \& Rydbeck, G. 2004, A\&A, 413, 505

MacArthur, L.A., Courteau, S., Bell, E., \& Holtzman, J., 2004, ApJS, 152, 175

Madau, P., Pozzetti, L., \& Dickinson, M. 1998, ApJ, 498, 106

McCall, M. L., Rybski, P. M., \& Shields, G. A. 1985, ApJS, 57, 1

Meurer, G. R., Heckman, T. M., \& Calzetti, D. 1999, ApJ, 521, 64 (M99)

Osterbrock, D. E. 1989 Astrophysics of Gaseous Nebulae and Active Galactic Nuclei (Mill Valley: University Science Books)

Pei, Y. C. 1992, ApJ, 395, 130

Popescu, C. C., Misiriotis, A., Kylafis, N. D., Tuffs, R. J., \& Fischera, J. 2000, A\&A, 362, 138

Popescu, C. C., Tuffs, R. J., Kylafis, N. D., \& Madore, B. F. 2004, A\&A, 414, 45

Popescu, C. C., et al. 2005, ApJ, 619, L75

Rice, W., 1993, AJ, 105, 67

van den Bergh, S. 1980, PASP, 92, 122

Witt, A. N., \& Gordon, K. D. 2000, ApJ, 528, 799 (WG00)

Witt, A. N., Thronson, H. A., \& Capuano, J. M. 1992, ApJ, 393, 611 